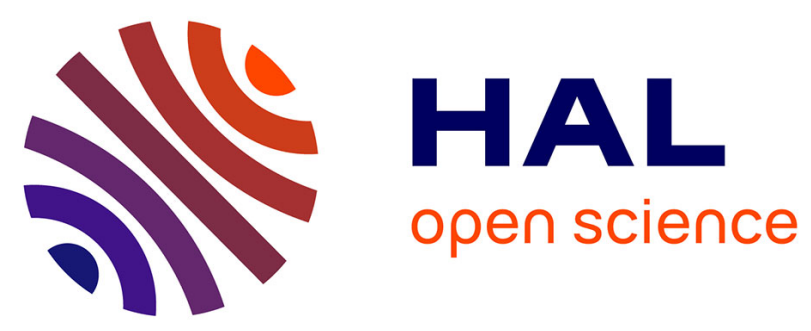

\title{
Asservissement d'un champ magnétique statique, dans la gamme 0,1 à 2 T, par R.M.N. impulsionnelle; optimalisation des performances
}

J. Alizon, J.P. Dagois, H. Robert

\section{- To cite this version:}

J. Alizon, J.P. Dagois, H. Robert. Asservissement d'un champ magnétique statique, dans la gamme 0,1 à 2 T, par R.M.N. impulsionnelle; optimalisation des performances. Revue de Physique Appliquée, 1979, 14 (3), pp.517-523. 10.1051/rphysap:01979001403051700 . jpa-00244622

HAL Id: jpa-00244622

https://hal.science/jpa-00244622

Submitted on 1 Jan 1979

HAL is a multi-disciplinary open access archive for the deposit and dissemination of scientific research documents, whether they are published or not. The documents may come from teaching and research institutions in France or abroad, or from public or private research centers.
L'archive ouverte pluridisciplinaire HAL, est destinée au dépôt et à la diffusion de documents scientifiques de niveau recherche, publiés ou non, émanant des établissements d'enseignement et de recherche français ou étrangers, des laboratoires publics ou privés. 


\title{
Asservissement d'un champ magnétique statique, dans la gamme 0,1 à $2 \mathrm{~T}$, par R.M.N. impulsionnelle ; optimalisation des performances
}

\author{
J. Alizon, J. P. Dagois et H. Robert \\ Laboratoire d'Electronique et Résonance Magnétique (*), \\ Université de Clermont II, B.P. 45, 63170 Aubière, France. \\ (Reçu le 13 juillet 1978, révisé le 7 novembre 1978, accepté le 8 novembre 1978)
}

\begin{abstract}
Résumé. - L'asservissement d'un champ magnétique statique, dans la gamme de 0,1 à $2 \mathrm{~T}$, a été construit et optimalisé par un choix convenable des temps de relaxation de l'échantillon capteur et par l'utilisation de séquences d'asservissement à multiples impulsions.

Des stabilités $\Delta B_{0} / B_{0}$ de $10^{-7}$ à $10^{-8}$ ont été obtenues dans toute la gamme de variation du champ.
\end{abstract}

\begin{abstract}
An automatic control of a magnetic static field, between 0.1 and $2 \mathrm{~T}$ was made. The performance was improved by using a reference sample with a convenient relaxation time and multiple pulses sequences. Stabilization factors $\Delta B_{0} / B_{0}$ of $10^{-7}$ to $10^{-8}$ was obtained in the whole range $0.1-2 \mathrm{~T}$.
\end{abstract}

1. Introduction. - L'asservissement par R.M.N. impulsionnelle étudié ici intervient en tant que troisième boucle d'asservissement du champ magnétique fourni par un électro-aimant BRUKER 100/100 SI 6n contrôlé à l'origine par une régulation d'intensité et un stabilisateur de flux. Cet ensemble équipe un spectromètre à impulsions SXP 4-100 de la même firme.

Dans la plupart des expériences en R.M.N. à champ variable (relaxation dipolaire, haute résolution dans les solides, accumulation de longue durée, etc...) il est nécessaire d'avoir des stabilités temporelles à long terme du champ magnétique très élevées

$$
\left(\Delta B_{0} / B_{0}=10^{-7} \text { à } 10^{-8}\right) .
$$

Par la régulation d'intensité seule, les valeurs

$$
\Delta B_{0} / B_{0} \simeq 10^{-4} \text { à } 10^{-5}
$$

sont atteintes à long terme.

Si le stabilisateur de flux permet d'obtenir des stabilités élevées à court terme, $\Delta B_{0} / B_{0} \simeq 10^{-7}$ à $10^{-8}$, et est très favorable pour l'insertion de l'asservissement par R.M.N. impulsionnelle, à long terme il ne fait que dégrader la stabilité obtenue par la régulation d'intensité.

(*) Equipe de recherche associée au C.N.R.S. n 90.
Par contre, l'asservissement par R.M.N. nous a permis d'atteindre les performances souhaitées dans la gamme de variation du champ magnétique de 0,1 à 2 teslas.

\section{Fonctions de transfert des boucles d'intensité et} de flux. - Avant d'entreprendre l'étude de l'asservissement par R.M.N. nous avons identifié de façon précise les différentes fonctions de transfert intervenant dans la régulation d'intensité et le stabilisateur de flux.

Nous avons ensuite simplifié les expressions de ces fonctions de transfert en introduisant les approximations justifiées par le fait que l'asservissement par R.M.N. n'intervient qu'en statique et basse fréquence. Ceci conduit à une expression très simple de la fonction de transfert en boucle fermée de la régulation d'intensité et du stabilisateur de flux et permet de s'affranchir de l'expression complexe de la fonction de transfert dynamique champ-courant.

2.1 FONCTION DE TRANSFERT DE LA RÉGULATION D'INTENSITÉ. - La figure 1 donne le schéma de principe de la régulation d'intensité et la figure 2 le schéma synoptique de fonctionnement qui permet d'obtenir l'expression de l'intensité $I[1]$ :

$$
I=\frac{V_{0}-G(p) U_{\mathrm{r}}}{[Z(p)+\rho]-G(p)[\rho+Z(p) T(p)]}
$$




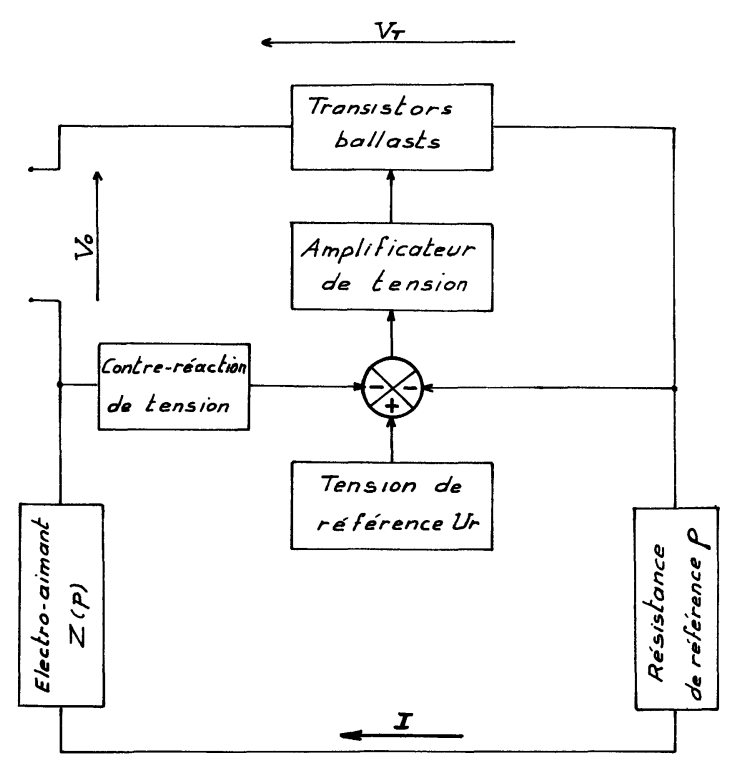

Fig. 1. - Schéma de principe de la régulation d'intensité.

[Diagram of the principle of the current regulation.]

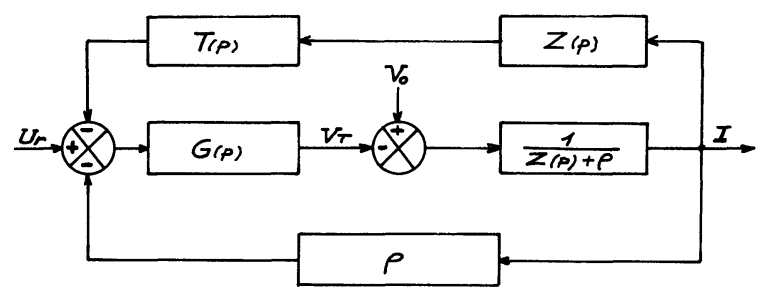

Fig. 2. - Synoptique de la régulation d'intensité.

[Scheme of the current regulation.]

avec :

$$
\begin{array}{l|l}
G(p)=G(0) \frac{1+A p}{1+B p} & \begin{array}{l}
G(0)=10^{6} \\
A=4,6 \mathrm{~s} \\
B=0,5 \mathrm{~s}
\end{array} \\
Z(p)=R+L p & \begin{array}{l}
R=0,6 \Omega \\
L=0,13 \mathrm{H}
\end{array} \\
T(p)=\frac{C p}{1+D p} & \begin{array}{l}
C=10^{-3} \mathrm{~s} \\
D=10^{-1} \mathrm{~s}
\end{array}
\end{array}
$$

$V_{0}$ : tension d'alimentation $\left(0<V_{0} \leqslant 120 \mathrm{~V}\right)$

$U_{\mathrm{r}}$ : tension de référence $\quad\left(0<U_{\mathrm{r}} \leqslant 1 \mathrm{~V}\right)$

$\rho$ : résistance de référence $\left(\rho \# 10^{-2} \Omega\right)$.

Compte tenu des valeurs numériques et du fait que l'asservissement par R.M.N. n'intervient qu'à très basse fréquence $(\$ 10 \mathrm{~Hz})$, l'expression de la fonction de transfert $J$ en boucle fermée de la régulation d'intensité s'écrit simplement :

$$
J=\frac{I}{U_{\mathrm{r}}}=\frac{1}{\rho} .
$$

2.2 FONCTION DE TRANSFERT DE LA RÉGULATION D'INTENSITÉ ET DU STABILISATEUR DE FLUX. - Les variations de flux magnétique sont captées à l'aide de bobines placées sur les pôles de l'électro-aimant. Elles permettent d'obtenir une tension proportionnelle à la dérivée du champ magnétique.

Cette tension est intégrée avant d'être appliquée à l'entrée de la boucle de régulation d'intensité comme le montre la figure 3 où $N$ et $S$ représentent respectivement le nombre de spires et la section des bobines captrices.

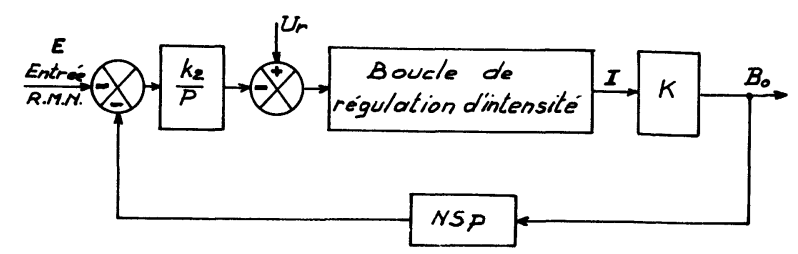

Fig. 3. - Synoptique de la régulation d'intensité et stabilisateur de flux.

[Scheme of the current regulation and flux stabilizer.]

Bien qu'en réalité l'intégrateur `soit réalisé à l'aide d'un amplificateur opérationnel de gain non infini (ce qui introduit alors une fonction de transfert du premier ordre, de constante de temps $\tau$, au lieu de $k_{2} / p$ ) l'analyse détaillée [1] montre que la représentation de la figure 3 est correcte pour l'étude des performances tant statiques que dynamiques de l'asservissement par R.M.N.

De plus, compte tenu des valeurs numériques, la fonction de transfert $F$ de la régulation d'intensité et du stabilisateur de flux s'écrit simplement :

$$
F=\frac{B_{0}}{E}=\frac{1}{N S p}=\frac{K_{1}}{p} ; \quad\left(K_{1}=\frac{1}{N S}\right) .
$$

Nous avons ainsi le comportement d'un intégrateur pur et nous remarquons que la fonction de transfert $K$ champ-courant $n$ 'intervient pas, éliminant ainsi les effets dus aux courants de Foucault, hystérésis, etc... difficilement contrôlables.

2.3 EfFicaCité dU STABIIISATEUR DE FlUX. - Pour juger de l'efficacité du stabilisateur de flux, nous avons défini le rapport $\eta$ tel que :

$$
\eta=\frac{\Delta B_{0}}{\Delta B_{0}^{\prime}} .
$$

$\Delta B_{0}$ et $\Delta B_{0}^{\prime}$ représentent respectivement les variations du champ magnétique avec et sans stabilisateur de flux pour une même perturbation appliquée sur les comparateurs de la boucle de courant et de flux.

Ce rapport $\eta$, fonction de la fréquence, fait intervenir les expressions dynamiques des différentes fonctions de transfert [1].

Les diagrammes asymptotiques de la variation de $\eta$ sont représentés sur la figure 4 et permettent de faire les remarques suivantes :

- L'efficacité maximale $\eta \simeq 10^{-3}$ se situe dans 


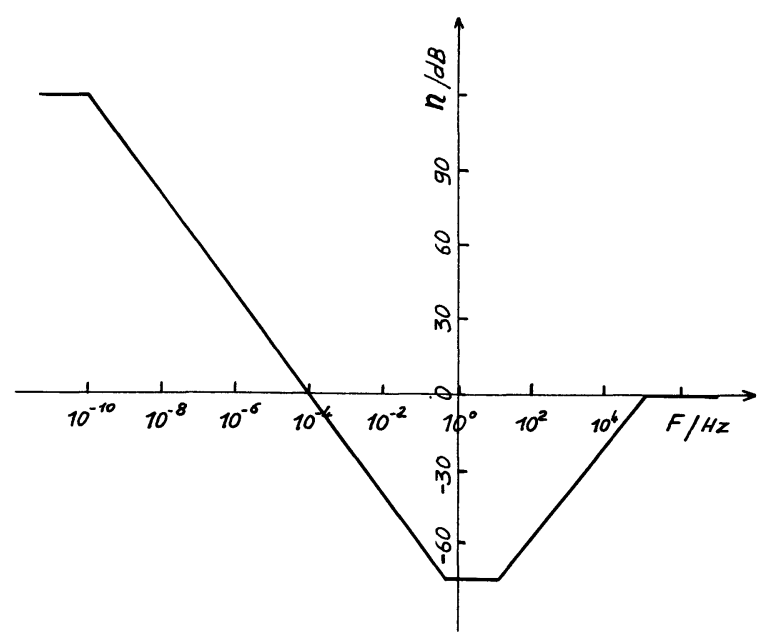

Fig. 4. - Efficacité $\eta$ du stabilisateur de flux en fonction de la fréquence.

[Frequency dependence of the efficiency $\eta$ of the flux stabilizer system.]

une gamme de fréquences comprise entre $10^{-1}$ et $10^{2} \mathrm{~Hz}$. Ceci est très favorable pour les corrections des perturbations dues au secteur et nous remarquons alors que la stabilité dynamique atteint la valeur $\Delta B_{0} / B_{0} \simeq 10^{-7}$ à $10^{-8}\left(10^{-4}\right.$ à $10^{-5}$ apporté par la régulation de courant et $10^{-3}$ par le stabilisateur de flux).

- Pour les fréquences inférieures à $10^{-4} \mathrm{~Hz}$ (c'est-à-dire à long terme) le stabilisateur de flux détériore la stabilité obtenue par la régulation d'intensité seule. Ceci est la conséquence du comportement en intégrateur non parfait (dérive des amplificateurs opérationnels...) de la boucle régulation d'intensité-stabilisateur de flux.

- Nous voyons alors la nécessité de la troisième boucle d'asservissement par R.M.N. qui permet de maintenir la stabilité $\Delta B_{0} / B_{0} \simeq 10^{-7}$ à $10^{-8}$ pour des fréquences inférieures à quelques hertz et surtout à très long terme.

\section{Asservissement par R.M.N. impulsionnelle. -} Le principe de base de l'asservissement du champ magnétique par R.M.N. impulsionnelle consiste à maintenir constante la période de battement $T_{\mathrm{B}}$ obtenue par détection synchrone d'un signal de référence à la pulsation $\omega_{\mathrm{r}}$ et du signal nucléaire de précession libre à la pulsation de Larmor $\omega_{0}=\gamma B_{0}$ [2], [3], [4], [5] (Figs. 5 et 6).

L'asservissement ainsi réalisé se comporte comme un système échantillonné. Le choix de la période du battement $T_{\mathrm{B}}$ (conditionné par l'inhomogénéité spatiale du champ magnétique) ainsi que celui de la période d'échantillonnage $T$ (fonction à la fois des temps de relaxation des échantillons principal et capteur) interviennent fortement dans le comportement du système.

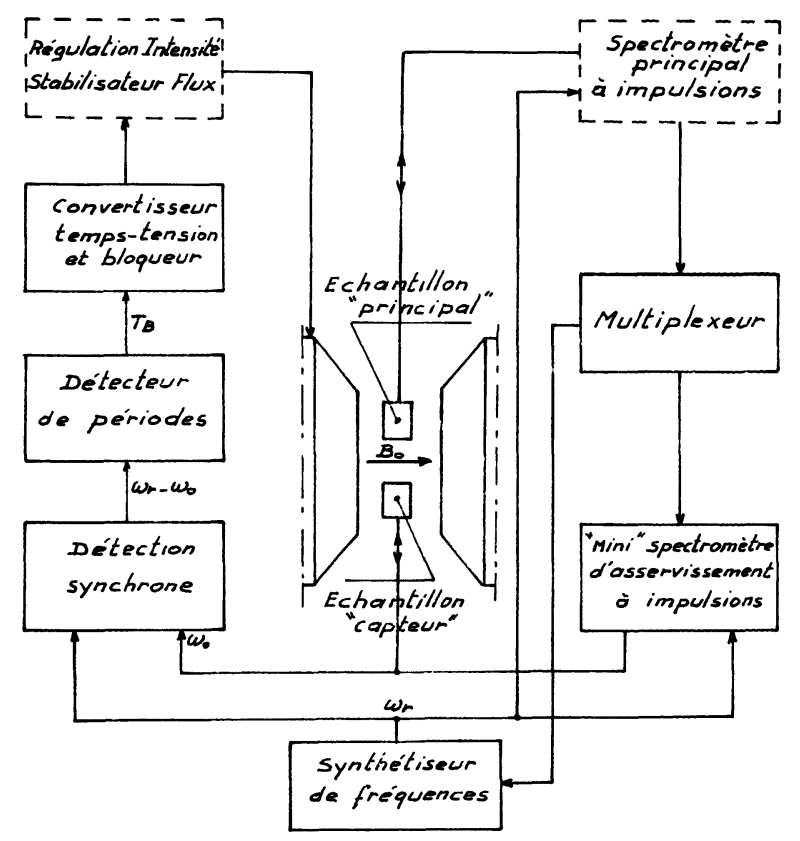

Fig. 5. - Synoptique de l'asservissement par R.M.N.

[Regulation system by R.M.N.]

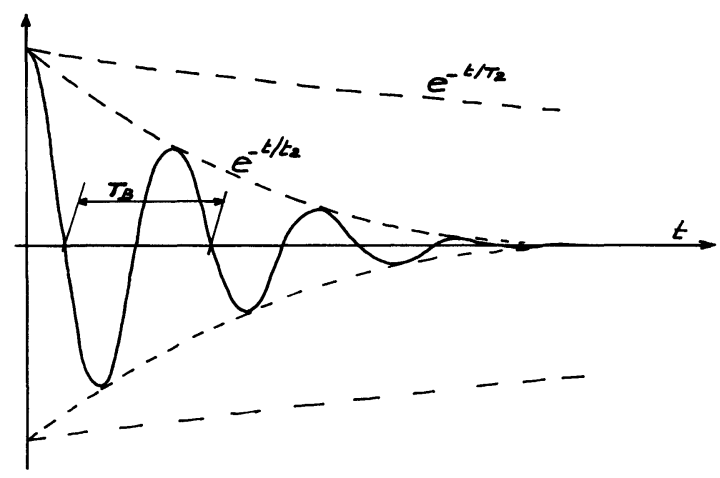

Fig. 6. - Signal de battement en sortie de la détection synchrone.

[Output beat signal from the lock-in detector.]

3.1 EXPRession De la STABILITÉ $\Delta B_{0} / B_{0}$. - Avec les notations définies ci-dessous,

$$
\begin{array}{ll}
\omega_{\mathrm{r}} & : \text { pulsation de référence } \\
\omega_{0}=\gamma B_{0} & : \text { pulsation de Larmor } \\
\Omega_{\mathrm{B}}=\omega_{\mathrm{r}}-\omega_{0} & : \text { pulsation du battement } \\
T_{\mathrm{B}}=\frac{2 \pi}{\Omega_{\mathrm{B}}} & : \text { période du battement }
\end{array}
$$

nous pouvons écrire :

$$
\begin{aligned}
\frac{\Delta B_{0}}{B_{0}} & =\frac{\Delta \omega_{0}}{\omega_{0}} \\
\Delta \omega_{0} & =\Delta \omega_{\mathrm{r}}-\Delta \Omega_{\mathrm{B}} \\
\Delta \Omega_{\mathrm{B}} & =-\frac{2 \pi}{T_{\mathrm{B}}} \cdot \frac{\Delta T_{\mathrm{B}}}{T_{\mathrm{B}}}
\end{aligned}
$$


soit, avec l'approximation $\omega_{\mathrm{r}} \# \omega_{0}$ :

$$
\frac{\Delta B_{0}}{B_{0}}=\frac{\Delta \omega_{\mathrm{r}}}{\omega_{\mathrm{r}}}+\frac{2 \pi}{\omega_{\mathrm{r}}} \frac{1}{T_{\mathrm{B}}} \frac{\Delta T_{\mathrm{B}}}{T_{\mathrm{B}}}
$$

Une stabilité élevée du champ magnétique exige que les deux termes du second membre soient très stables :

- Pour le premier $\Delta \omega_{\mathrm{r}} / \omega_{\mathrm{r}}$, il n'y a pas de problème car la pulsation $\omega_{\mathrm{r}}$ est fournie par un synthétiseur de fréquence pour lequel on a couramment

$$
\Delta \omega_{\mathrm{r}} / \omega_{\mathrm{r}} \simeq 10^{-9}
$$

à long terme.

- Le deuxième terme, par contre, exige une analyse plus approfondie. On peut remarquer qu'il sera d'autant plus élevé que la pulsation de travail $\omega_{\mathrm{r}}$, ou le champ magnétique, sera plus faible ce qui laisse prévoir des difficultés d'asservissement à bas champ. Ensuite intervient le facteur $1 / T_{\mathrm{B}}$; il suffirait, a priori, de choisir $T_{B}$ le plus grand possible pour rendre ce deuxième terme négligeable et à la limite on aurait $\Delta B_{0} / B_{0} \simeq \Delta \omega_{\mathrm{r}} / \omega_{\mathrm{r}}$. Nous sommes cependant limités dans cette voie pour deux raisons principales :

a) l'inhomogénéité spatiale du champ magnétique,

b) la stabilité (accrochage) et la bande passante de l'asservissement par R.M.N.

3.2 INFLUENCE DE L'INHOMOGÉNÉITÉ DE L'AIMANT. - L'inhomogénéité spatiale $\Delta b_{0}$ du champ magnétique sur le volume de l'échantillon est caractérisée par un temps $T_{2}^{*}\left(T_{2}^{*}=2 / \gamma \Delta b_{0}\right)$ qui se traduit par une décroissance plus rapide (Fig. 6) du signal nucléaire de précession libre en un terme $t_{2}$ tel que [1] :

$$
\frac{1}{t_{2}}=\frac{1}{T_{2}}+\frac{1}{T_{2}^{*}}
$$

avec $T_{2}$, temps de relaxation spin-spin.

Afin de pouvoir mesurer la période du battement $T_{\mathrm{B}}$ avec précision tout en conservant une valeur élevée de $T_{\mathrm{B}}$, nous nous imposons de prendre $T_{\mathrm{B}} \simeq t_{2}$ (Fig. 6).

3.3 StABILITÉ DE L'ASSERVISSEMENT. - L'asservissement par R.M.N. impulsionnelle se comporte comme un système échantillonné de période $T$.

Le schéma synoptique de la figure 7 permet l'étude de la stabilité (accrochage) et fait intervenir les fonctions de transfert :

- du convertisseur temps-tension $K_{2}$,

- du bloqueur d'ordre zéro $Z_{0}(p)$,

- de la régulation d'intensité-stabilisateur de flux $F=K_{1} / p$,

- de la boucle de retour, par le rapport gyromagnétique $\gamma$.

La fonction de transfert échantillonné

$$
R(z)=B_{0}(z) / \omega_{\mathbf{r}}(z)
$$

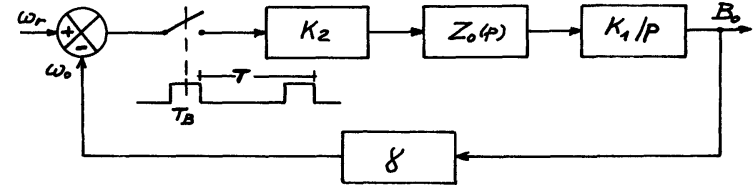

Fig. 7. - Synoptique, pour l'étude de la stabilité, de l'asservissement par R.M.N. impulsionnelle.

[Study of the stability : scheme of the regulation system by pulsed R.M.N.]

s'écrit, avec $K_{0}=K_{1} K_{2}$ :

$$
R(z)=\frac{K_{0}\left[Z_{0}(p) / p\right](z)}{1+K_{0} \gamma\left[Z_{0}(p) / p\right](z)}
$$

Le système est stable lorsque l'équation caractéristique $\left\{1+K_{0} \gamma\left[Z_{0}(p) / p\right](z)\right\}=0$ ne possède aucun zéro à l'intérieur du cercle unité dans le plan $z$ [5] avec $z=x+j y$ (Fig. 8), or,

$\left[\frac{Z_{0}(p)}{p}\right](z)=\frac{T}{z-1} \quad(T:$ période d'échantillonnage $)$ d'où $z-1+K_{0} \gamma T=0$ soit $x=1-K_{0} \gamma T$.

Le lieu représentatif de cette équation caractéristique est situé sur l'axe des réels et, pour assurer la stabilité, nous devons avoir $x>-1$, soit

$$
K_{0} \gamma<\frac{2}{T}
$$

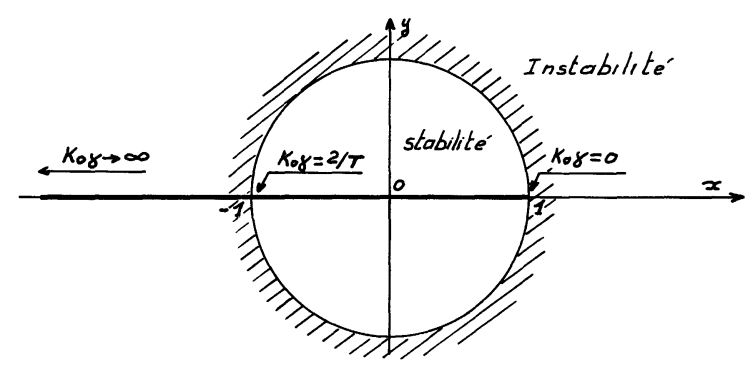

Fig. 8. - Lieu des racines de l'équation caractéristique $z-1+K_{0} \gamma T=0$ dans le plan $z=x+j y$.

[Root locus of the characteristic equation $z-1+K_{0} \gamma T=0$ in the plane $z=x+j y$.]

La figure 9 représente $K_{0} \gamma$ en fonction de la période d'échantillonnage $T$.

Remarque. - L'utilisation du stabilisateur de flux est très favorable au fonctionnement de l'asservissement par R.M.N. car il permet d'introduire un pôle dans la fonction de transfert de la chaîne principale ce qui rend nulle l'erreur stationnaire $\varepsilon_{01}$ du premier ordre.

En effet, $\varepsilon_{01}=\omega_{\mathrm{r}}-\omega_{0}$

$$
\varepsilon_{01}=\lim _{z \rightarrow 1} \frac{z-1}{z} \frac{\omega_{\mathrm{r}}(z)}{1+K_{0} \gamma\left[Z_{0}(p) / p\right](z)} .
$$




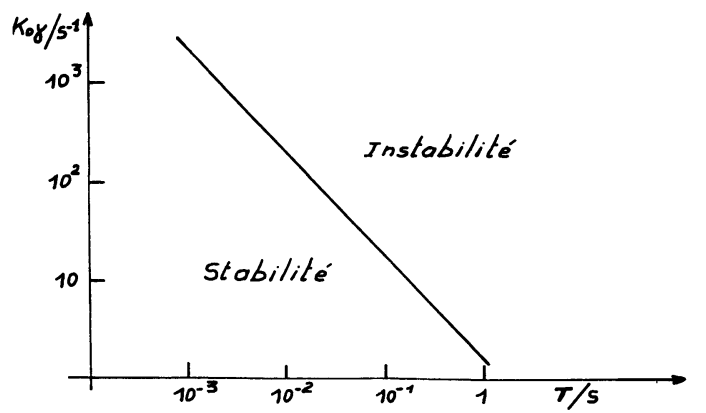

Fig. 9. - Représentation du gain en boucle $K_{0} \gamma$ en fonction de la période d'échantillonnage $T$, à la limite de stabilité de l'asservissement.

[Gain loop $K_{0} \gamma$ as a function of the period of sampling $T$, at the limit of the regulation system stability.]

Or

$$
\omega_{\mathrm{r}}(z)=\omega_{\mathrm{r}} \cdot \frac{z}{z-1}
$$

et

$$
\left[\frac{Z_{0}(p)}{p}\right](z)=\frac{T}{z-1}
$$

d'où

$$
\begin{aligned}
\varepsilon_{01} & =\lim _{z \rightarrow 1} \frac{z-1}{z} \omega_{\mathrm{r}} \frac{z}{z-1} \frac{1}{1+K_{0} \gamma T /(z-1)} \\
\varepsilon_{01} & \rightarrow 0 .
\end{aligned}
$$

3.4 BANDE PASSANTE DE L'ASSERVISSEMENT. - Nous allons donner l'expression de la bande passante de l'asservissement par R.M.N. vis-à-vis des principales perturbations que nous supposerons appliquées à l'intérieur de la boucle et à l'entrée de la chaîne régulation d'intensité-stabilisateur de flux selon le schéma de la figure 10.

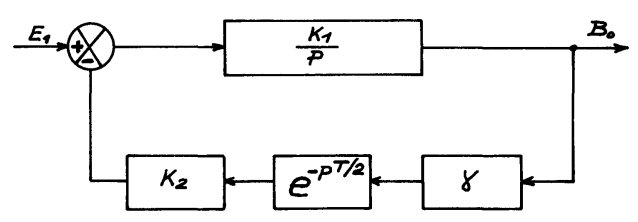

Fig. 10. - Synoptique, pour l'étude de la bande passante, de l'asservissement par R.M.N. impulsionnelle.

[Study of the bandwidth : scheme of the regulation system by pulsed R.M.N.]

D'autre part, pour cette étude, nous assimilerons l'échantillonneur-bloqueur d'ordre zéro à un retard pur [6] de fonction de transfert $\exp (-p T / 2)$.

La fonction de transfert $G=B_{0} / E_{1}$ s'écrit :

$$
G(p)=\frac{K_{1}}{p+K_{0} \gamma \mathrm{e}^{-p T / 2}}
$$

soit, avec $p=j \omega$ et $K_{0}=K_{1} K_{2}$ :

$$
G(\omega)=\frac{K_{1}}{K_{0} \cos \omega T / 2+j\left(\omega-K_{0} \gamma \sin \omega T / 2\right)} .
$$

La pulsation de coupure $\omega_{\mathrm{c}}$, définie par $0 \mathrm{~dB}$ par rapport à $G(0)$, est telle que :

$$
|G(0)|=\left|G\left(\omega_{\mathrm{c}}\right)\right|
$$

soit :

$$
2 \sin \omega_{\mathrm{c}} T / 2=\omega_{\mathrm{c}} / K_{0} \gamma .
$$

En choisissant le gain $K_{0} \gamma$ tel que le système soit à la limite de stabilité, c'est-à-dire $K_{0} \gamma=2 / T$, nous obtenons la relation :

$$
2 \sin \omega_{\mathrm{c}} T / 2=\omega_{\mathrm{c}} T / 2 .
$$

Cette équation est de la forme $(\sin X) / X=1 / 2$ et a pour solution $X \# 3 \pi / 5$ soit

$$
\omega_{\mathrm{c}} \# \frac{4}{T} \text {. }
$$

La fréquence de coupure de l'asservissement par R.M.N. impulsionnelle est ainsi inversement proportionnelle à la période de répétition des impulsions d'asservissement.

4. Optimalisation des performances. - Les performances de l'asservissement dépendent principalement :

- des temps de relaxation $T_{1}$ et $T_{2}$ de l'échantillon capteur,

- de la période $T_{\mathrm{B}}$ du signal d'asservissement,

- de la période $T$ d'échantillonnage.

Ces paramètres doivent être choisis en fonction des valeurs du champ magnétique et du temps de relaxation spin-réseau $T_{1}^{\prime}$ de l'échantillon principal.

4.1 ChoIX DE LA Période $T$ D'éChantillonNage. - Pour accroître les performances dynamiques de l'asservissement par R.M:N., nous devons choisir la période d'échantillonnage la plus faible possible. Toutefois, par principe même de l'asservissement, cette période $T$ doit rester supérieure ou de l'ordre de grandeur de la période du battement $T_{\mathrm{B}}$.

De plus, pour obtenir un signal d'asservissement d'amplitude maximale, nous devons également choisir la période $T$ du même ordre de grandeur que le temps de relaxation spin-réseau $T_{1}$ de l'échantillon capteur.

L'influence du temps de relaxation $T_{1}^{\prime}$ de l'échantillon principal se manifeste de la façon suivante :

$1^{\text {er }}$ cas : $T_{1}^{\prime}$ est court $\left(T_{1}^{\prime} \leqslant 0,1 \mathrm{~s}\right)$. - Nous choisissons $T \simeq T_{1}^{\prime}$ et une seule impulsion d'asservissement par séquence (Figs. 11a,b).

La bande passante de l'asservissement par R.M.N. est alors supérieure ou de l'ordre de $10 \mathrm{~Hz}$.

Remarques. - Les impulsions d'asservissement sont synchrones des impulsions provenant du spectromètre principal. 


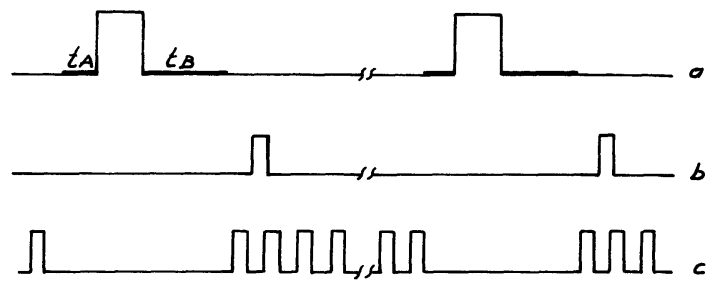

Fig. 11. - $a$ ) Définition des temps $t_{\mathrm{A}}$ et $t_{\mathrm{B}}$ par rapport à l'impulsion du spectromètre principal. $b$ ) Séquence d'asservissement simple impulsion. c) Séquence d'asservissement multiples impulsions.

[a) Definition of the times $t_{\mathrm{A}}$ and $t_{\mathrm{B}}$ versus the pulse of the main spectrometer. $b$ ) Sequence of regulation simple pulse. c) Sequence of regulation multiple pulses.]

- Un dispositif électronique a également été conçu [1] pour interdire l'application des impulsions d'asservissement pendant les temps $t_{\mathrm{A}}$ et $t_{\mathrm{B}}$ (Fig. 11a) nécessaires aux acquisitions respectives des signaux d'asservissement et des signaux issus du spectromètre principal.

$2^{\mathrm{e}}$ cas : $T_{1}^{\prime}$ est long $\left(T_{1}^{\prime}>0,1 \mathrm{~s}\right)$. - Afin de conserver les performances dynamiques du premier cas, nous utilisons alors une séquence multiples impulsions d'asservissement (Figs. 11a,c).

La période d'échantillonnage est alors choisie la plus faible possible tout en respectant la condition $T>T_{\mathrm{B}}$.

4.2 Choix De la période $T_{\text {B }}$ Des battements. Les performances statiques élevées de l'asservissement par R.M.N. imposent de choisir la période du signal d'asservissement la plus élevée possible.

Cependant, l'inhomogénéité spatiale du champ magnétique limite la valeur de $T_{\mathrm{B}}$ à $T_{2}^{*}$.

En effet, comme nous l'avons vu au paragraphe 3.2, nous avons choisi $T_{\mathrm{B}} \simeq t_{2}$ avec $1 / t_{2}=1 / T_{2}+1 / T_{2}^{*}$.

Pour un échantillon capteur à temps de relaxation élevé $\left(T_{2} \gg T_{2}^{*}\right)$ nous avons bien $t_{2} \# T_{2}^{*}$.

4.3 CHOIX DE L'ÉCHANTILLON " CAPTEUR " ET DE SES TEMPS DE RELAXATION. - Aux paragraphes 4.1 et 4.2 , nous avons établi les conditions suivantes :

$$
\begin{aligned}
T & \geqslant T_{\mathrm{B}} \\
T & \simeq T_{1} \\
T_{\mathrm{B}} & \simeq t_{2} .
\end{aligned}
$$

Pour parfaire l'optimalisation de l'asservissement, nous devons choisir l'échantillon capteur tel qu'il permette d'obtenir la période d'échantillonnage la plus courte possible et la période du battement la plus élevée possible en donnant la priorité à cette dernière car c'est elle qui conditionne une bonne stabilité du champ magnétique à long terme.

4.3.1 Echantillon « capteur » solide. - Dans ce cas, où généralement $T_{2} \ll T_{2}^{*} \ll T_{1}$, les deux conditions sur les périodes d'échantillonnage $T$ et du battement $T_{\mathbf{B}}$ ne sont pas réalisées (Fig. 12a).
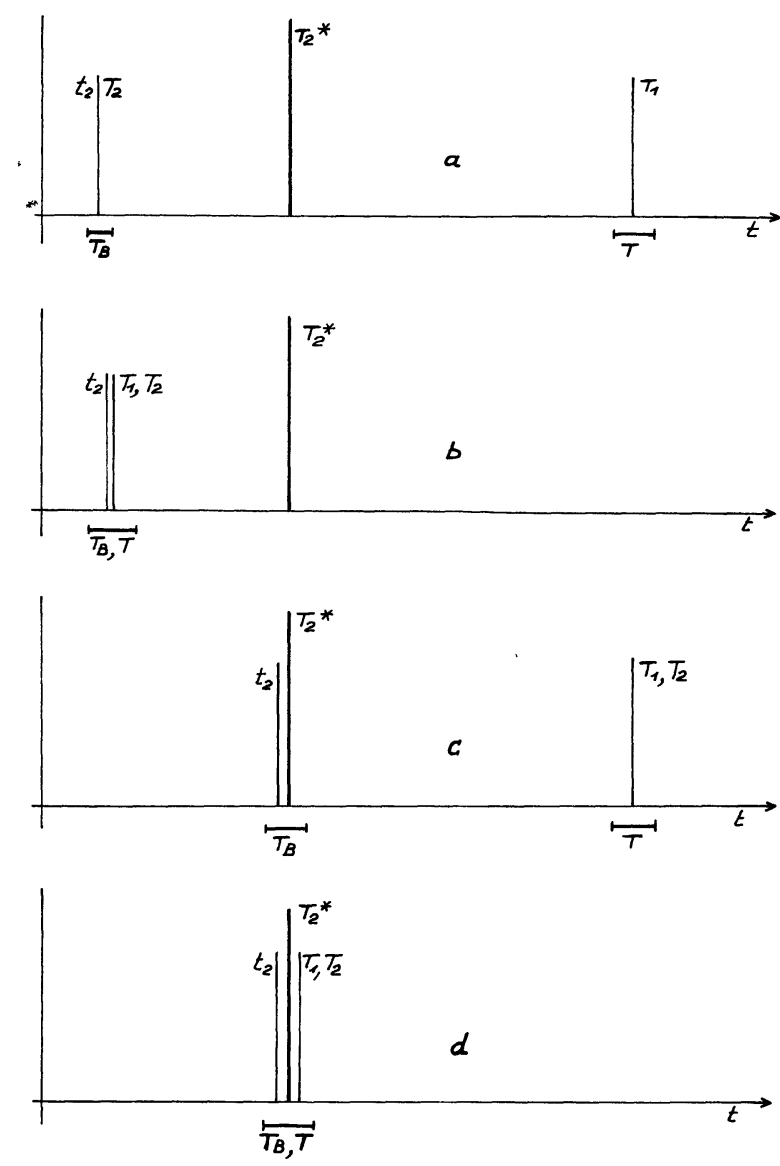

Fig. 12. - a) Echantillon capteur solide. $b, c$ ) Echantillon capteur liquide mal choisi. $d$ ) Echantillon capteur bien choisi.

(a) Solid probe. $b, c$ ) A badly adapted liquid probe. d) A well adapted liquid probe.]

En effet :

$$
\begin{array}{cl}
T \geqslant T_{1} & \text { serait élevé } \\
T_{\mathrm{B}} \simeq t_{2} \# T_{2} & \text { serait faible } .
\end{array}
$$

4.3.2 Echantillon "capteur» liquide. - Pour un échantillon liquide où $T_{1} \# T_{2}$, trois situations peuvent se présenter :

$1^{\text {er }}$ cas : $T_{1}=T_{2} \ll T_{2}^{*}$. - Dans ce cas, la condition sur la période d'échantillonnage est bien satisfaite mais pas celle sur la période des battements (Fig. 12b).

$2^{\mathrm{e}}$ cas : $T_{1}=T_{2} \gg T_{2}^{*}$. - Ici la condition sur la période des battements est satisfaite, mais pas celle sur la période d'échantillonnage (Fig. 12c).

En effet :

$$
\begin{array}{cl}
T_{\mathrm{B}} \simeq t_{2} \# T_{2}^{*} & \text { serait satisfait } \\
T \geqslant T_{1} & \text { serait élevé } .
\end{array}
$$

3e cas : $T_{1}=T_{2} \# T_{2}^{*}$. - Cette situation correspond à un échantillon capteur convenablement choisi car ses temps de relaxation permettent de satisfaire les conditions requises pour obtenir à la fois de bonnes performances dynamiques et statiques de l'asservissement par R.M.N. impulsionnelle. 
Nous obtenons alors les conditions très simples à satisfaire, imposées par l'inhomogénéité spatiale du champ magnétique caractérisé par le temps $T_{2}^{*}$ (Fig. 12d) :

$$
T \simeq T_{1}=T_{2} \simeq T_{2}^{*} \simeq T_{\mathrm{B}}
$$

rappelons que :

- $T$ est la période d'échantillonnage,

- $T_{1}, T_{2}$ sont les temps de relaxation de l'échantillon capteur,

- $T_{2}^{*}$ est le temps caractéristique de l'inhomogénéité du champ,

- $T_{\mathrm{B}}$ est la période des battements du signal d'asservissement.

L'asservissement du champ magnétique à large bande $(0,1$ à $2 \mathrm{~T})$, où $T_{2}^{*}$ est fonction décroissante du champ, impose de changer d'échantillon capteur en fonction de la valeur du champ affiché pour satisfaire les conditions ci-dessus.

Pour notre appareillage, $T_{2}^{*}$ varie de $10 \mathrm{~ms}$ à $0,5 \mathrm{~ms}$ environ quand le champ passe de 0,1 à $2 \mathrm{~T}$ et nous utilisons de l'eau convenablement dopée en nitrate ferrique pour l'échantillon capteur.

5. Performances obtenues. Conclusion. - Nous avons contrôlé la stabilité $\Delta B_{0} / B_{0}$ du champ magnétique à l'aide d'une chaîne de mesure construite [1] sur le même principe que la chaîne d'asservissement, avec en plus un circuit de linéarisation permettant d'avoir une tension directement proportionnelle à $\Delta B_{0} / B_{0}$ (Fig. 13).

Le facteur d'amélioration des dérives à long terme, par rapport à la régulation d'intensité seule, est voisin de 1000 dans toute la gamme de variation du champ magnétique de 0,1 à 2 teslas. La figure 14 donne un exemple d'enregistrement des variations de $\Delta B_{0} / B_{0}$ en fonction du temps.

Des stabilités $\Delta B_{0} / B_{0} \simeq 10^{-8}$ sont atteintes quasi instantanément sur un changement de la valeur $\mathrm{du}$ champ, contrairement à la régulation d'intensité qui demande environ une journée de fonctionnement pour obtenir, à seulement $10^{-5}$ près, la valeur désirée du champ.

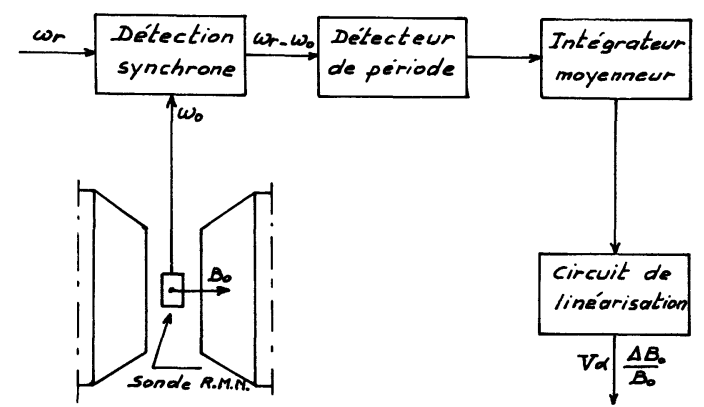

Fig. 13. - Chaîne de mesure pour le contrôle de la stabilité $\Delta B_{0} / B_{0}$ du champ magnétique.

[Measuring set for the control of the magnetic field stability $\Delta B_{0} / B_{0}$.]

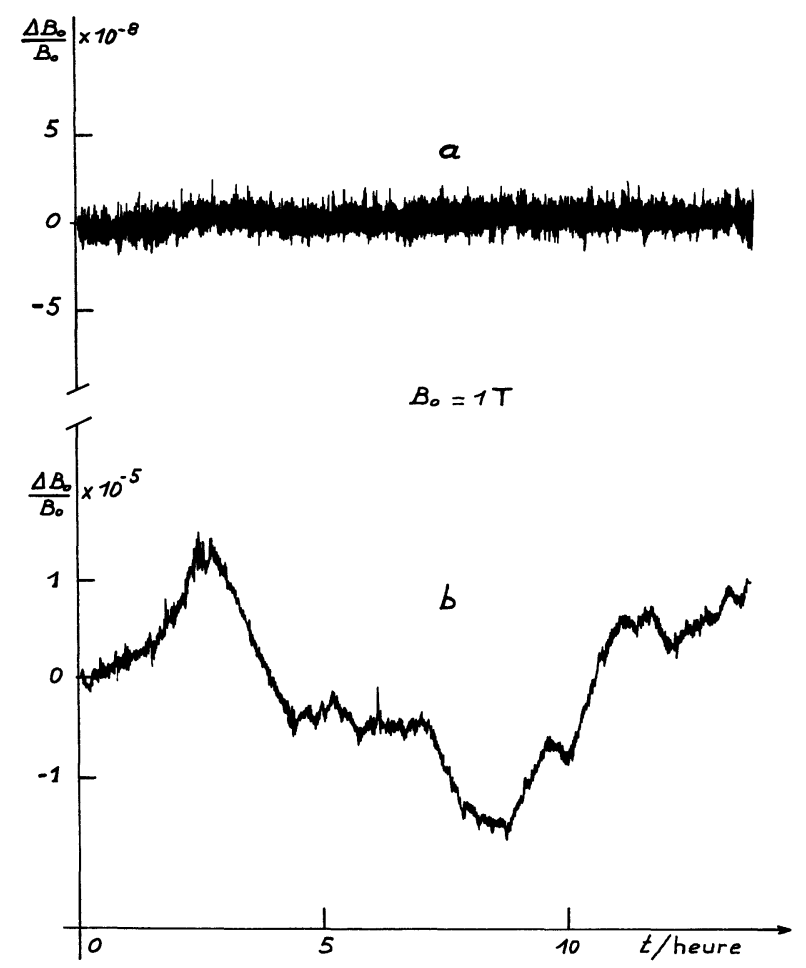

Fig. 14. - Enregistrement de $\Delta B_{0} / B_{0}$ en fonction du temps : a) avec asservissement par R.M.N. impulsionnelle; b) avec la régulation d'intensité seule.

[Recording of $\Delta B_{0} / B_{0}$ as a function of time : a) regulation system by pulsed R.M.N.; $b$ ) current regulation only.]

\section{Bibliographie}

[1] Dagois, J. P., Thèse de $3 \mathrm{e}$ cycle, Clermont-Ferrand (1978).

[2] Block, F., Phys. Rev. 70 (1946) 460.

[3] Hahn, E. L., Phys. Rev. 80 (1950) 580.

[4] Alizon, J., Thèse d'Etat, Clermont-Ferrand (1974).
[5] Bruker Spectrospin, S. A., Notice B-SN 15.

[6] Y. Sevely. - Systèmes et asservissements linéaires échantillonnés (Dunod) 1972. 